\title{
Hands-on training in dynamic navigation
}

Implant dentists who want to take precision and safety to a new level can learn how with Master Clinical Trainer, Dr David Burgess. Now in its fourth year, the Dynamic Navigation Society's UK training programme includes regular one-day introductory courses in freehand surgery using real-time computer guidance. Delegates are given an overview of the complete process from patient diagnosis to navigated implant treatment. Bespoke two-day masterclasses offer training specifically tailored to the attendee's requirements.

Participants have the opportunity to prepare dental implant sites on models and phantom heads with dynamic navigation technology. They observe case planning

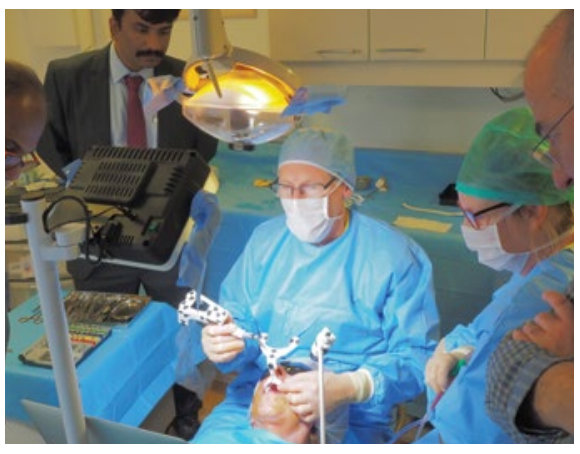

and live surgery using the Navident system, learning about the digital dentistry workflow and clinical protocol.

The two-day masterclass is aimed at dentists who already use dynamic navigation and want to accelerate integration into their everyday practice. The course provides a comprehensive insight into the benefits of freehand surgery with real-time computer guidance.

Details of the latest courses can be obtained by calling 0845602 4944, emailing info@dynamicnavigationsociety.org.uk or at http://bit.ly/DNSUKcourses. Courses are limited to five delegates for maximum benefit. Early bird discounts apply.

The one-day introductory course will be held on 9 August, 20 September and 1 November 2019, then on 28 February, 3 April and 12 June 2020. The two-day masterclasses can be arranged at any mutually convenient time. Courses are held at Carbis Bay Dental Care, Cornwall.

\section{Predictable excellence}

A patient experiencing pain and in need of endodontic treatment might still be reluctant to go ahead.

Root canal therapy has a bad reputation: that it is painful and unpleasant. Reassure your patients by only using the very best tools and materials. COLTENE has a comprehensive range of premium products, from rotary files to sealing materials, which facilitate the treatment pathway and help to ensure predicable, stable results.

COLTENE is the mark of excellence.
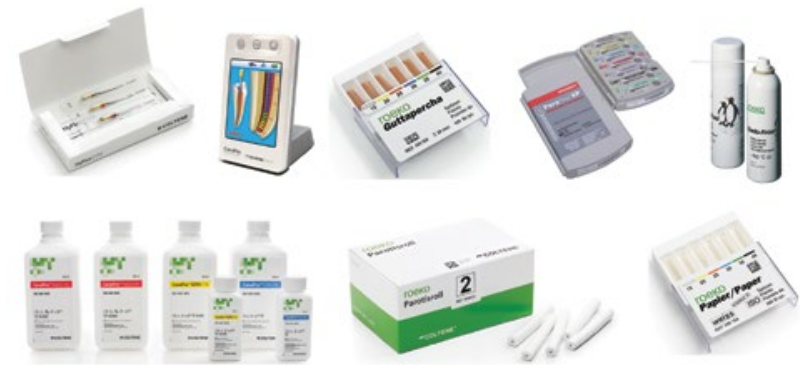

When you use COLTENE products in your endodontic work, you can assure nervous patients of a successful treatment that will improve their quality of life. They should accept nothing less - and neither should you!

To find out more visit www.coltene.com, emailinfo.uk@coltene.com or call 01444 235486.

\section{As fast as it is smart}

Dürr Dental have developed an extended version of their VistaVox S panoramic machine which contains six additional programmes for time-saving cephalometric exposure with minimum radiation doses, called VistaVox S Ceph.

As you'd expect from Dürr, exceptional diagnostics and ease of use are guaranteed. Alongside the 17 panoramic programmes, the VistaVox S Ceph has several orthodontic applications, including 'Lateral Head', 'Full Lateral Head', 'PA Head' and 'Waters View'. The unit is as fast as it is smart - with a scan time of just 1.9 seconds, images are exceptionally sharp using the lowest possible radiation dose. This functionality is afforded by the high-sensitivity CSL sensors. The unit can effortless switch between the 3D X-Ray and the Ceph boom, a process that on some machines can be both cumbersome and risky.

Just like the VistaVox $S$ it has a perfect $3 \mathrm{D}$ imaging volume of $130 \mathrm{~mm}$ (compared to $80 \mathrm{x} 80 \mathrm{~mm}$ for most other systems). This means it completely covers the whole diagnostically relevant area, including the rear molars, an essential requirement for diagnosing an impacted wisdom tooth. Enhanced visibility does not require a higher radiation dose; in fact, the opposite is true. A special curved path, which rotates $540^{\circ}$, in combination with a tightly collimated fan beam and a highly sensitive Csl sensor, means that a particularly low radiation dose is used.

Similarly to the VistaVox $S$, this enhanced model offers $\varnothing 50 \times 50 \mathrm{~mm}$ volumes, for indications that only require a certain part

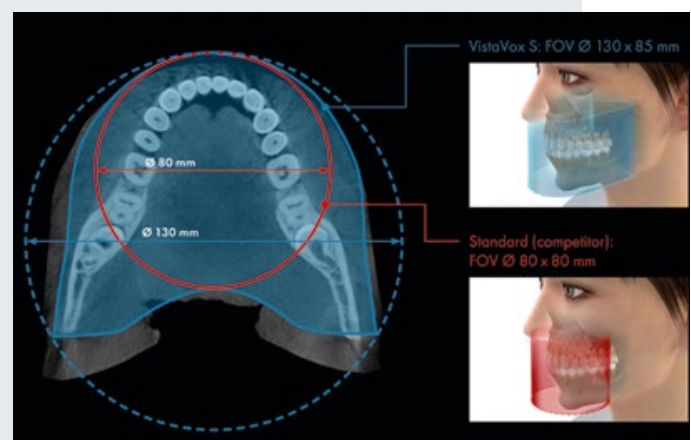

of the jaw region to be shown, eg for endodontic or implant treatments. The unit offers true all-in-one capabilities for a full range of diagnostics making it ideal for dentists, orthodontists or those who work within larger practices offering a full range of specialist treatments. 